\title{
The Impact of the Increasing Popularity of Digital Art on the Current Job Market for Artists
}

\author{
Vivian Wang1, Dali Wang2 \\ ${ }^{1}$ Farragut Club of Digital Arts and Coding, Kingston Pike, Farragut, USA \\ ${ }^{2}$ Department of Electrical Engineering and Computer Science, University of Tennessee, Knoxville, USA \\ Email: vivian.wang0109@hotmail.com, dwang7@utk.edu
}

How to cite this paper: Wang, V., \& Wang, D. (2021). The Impact of the Increasing Popularity of Digital Art on the Current Job Market for Artists. Art and Design Review, 9, 242-253.

https://doi.org/10.4236/adr.2021.93019

Received: May 25, 2021

Accepted: July 19, 2021

Published: July 22, 2021

Copyright $\odot 2021$ by author(s) and Scientific Research Publishing Inc. This work is licensed under the Creative Commons Attribution International License (CC BY 4.0).

http://creativecommons.org/licenses/by/4.0/

\begin{abstract}
Digital art has become a part of daily life. Despite the rising popularity of digital art and its advantages over traditional art, it is still unclear what the impact digital art has on the job market for artists today. This research provided a practical and quantitative method to investigate the impact of the rising popularity of digital art on the national job market for artists today. This study collected and sampled online data across the US and performed quantitative analysis. This study shows, in the study period (January-April, 2021), around 22,500 artist jobs available, and over 1\% (2400) of them are high-paid jobs with an annual salary of over $100 \mathrm{~K}$, and over $3 \%$ (7515) of them are medium-paid jobs with a salary of over $65 \mathrm{~K}$. The majority (66\%) of artist jobs with an annual salary of over $60 \mathrm{~K}$ are related to digital art and come from technology sectors. The digital art jobs are across the nation, not clustered around major cities.
\end{abstract}

\section{Keywords}

Digital Art, Job Market, Artist Job Functions, Artist Job Distribution, Digital Artist Salary

\section{Introduction}

More and more types of digital art emerge as technology advances. Today, digital art has become a part of daily life and greatly impacts traditional art (Schukei, 2019; Sanders, 2018), although many digital artists started learning traditional art. Compared to traditional art, the most significant advantage of digital art is its convenience: digital art is easy to carry, easy to publish and share, easy to be printed in many places, and most importantly, easy to be corrected. Digital art also has the edge over traditional art, considering its economic cost and potential 
incomes. Most recently, a piece of digital artwork that does not exist in physical form was sold at 69 million dollars. Despite the rising popularity of digital art and its advantages over traditional art, it is still unclear what the impact digital art has on the job market for artists today. Historical data and artist job outlooks do not show a rosy picture. However, many articles presented optimistic views towards digital artists. In this study, I would like to design my method to collect data on job markets and to investigate the impact of the rising popularity of digital art on the job market for artists today. Specifically, I would like to investigate the job market for digital artists, including the size, salary information, job functions, and demographic distributions of these jobs.

\section{Literature Review}

\subsection{History of Digital Art}

In this study, digital art is defined as an artistic work or practice that uses digital technology as part of the creative or presentation process (Paul, 2008). Digital art started in the 1960s as a new form of media art (Agyeman, 2014; Edmonds, 2019; Heck, 2017). Some artists and historians think that digital art is a new way of using machines to create art. They tracked back the starting period of digital art to the beginning of the 20th century (Bessette, 2018).

\subsection{Types of Digital Art}

More and more types of digital art emerge as technology advances. In a featured article (Anton, 2020), the artists from Architecture Lab provide a comprehensive review of 15 common types of digital art. Some of them are common in our life, such as 2D Computer Graphics, Digital Photography, Photo-painting, 3D Computer Graphics, Pixel Art, Digital Photography, Digital Collage, 2D Digital Painting, and 3D Digital Painting.

Digital art has its characters and is significantly different from traditional art (Zagrobelna, 2017). Digital art can be viewed as the same, just binary code shown visually on a screen. However, digital art is usually done on a drawing tablet and with a stylus. The stylus can act as any tool, brush, blender, or pencil. One unique thing traditional art cannot do is animation, displaying an image/ drawing every fraction of a second to create movement. Digital art also has layers, blend modes, clipping masks, paint bucket tools, resizing, reshaping, and magic rulers. These digital-specific tools allow for unique special effects and a completely new process for creation when it comes to creating art.

\subsection{Future of Digital Art}

The rise of digital art has a significant impact on traditional art; it also draws criticism (Heck, 2017). Many critics and curators think that tools create digital artworks, and they do not want to acknowledge the artistry of the digital art creators. However, the wide acceptance of digital art is inevitable, just like many other forms of art in the past. There are reasons to be optimistic about the future 
of digital art (Agyeman, 2014; Butcher, 2017; Didenko, 2018). First, digital tools are revolutionizing the transformation of the digital arts in the film industry. Second, museums around the world are starting to accept digital illustrations and portraits. Finally, digital artworks have become an integral part of social life, such as fashion or interior design.

The advantages of digital art are apparent. Many artists started learning traditional art when they were young but have gradually spent more and more time on digital art. These artists shared similar opinions with Kat Sander (Sanders, 2018), that the biggest advantage of digital art is its convenience. The artists can bring the iPad anywhere and not worry about any extra overspending. It is easy to publish and share the works via social media once they are done. The works can be printed in many places: shirts, mugs, and so on. Most importantly, it is easy to correct any mistakes during the process. Digital art is also more viable than traditional art considering economic cost and potential incomes.

\subsection{Job Market Outlook and Job Income}

Despite the rising popularity of digital art and its advantages over traditional art, it is still unclear what the impact digital art has on the job market for artists today. Historical data from 2008 to 2018 showed the overall artist-employment growth rate is around 10\% (Iyengar, 2011). More specifically, around a $25 \% \mathrm{em}$ ployment increase is expected for these occupants, such as museum technicians and conservators, curators, landscape architects, and interior designers. While architects, writers, and actors are projected to increase at the average rate of $18 \%$. The artist occupations: radio and television announcers, fashion designers, and floral designers expect a below $5 \%$ increase. Also, based on the Bureau of Labor Statistics' labor report for 2019-2029, the author listed four art careers: art director, fashion designer, graph designer, multimedia artists, and animators (Writers, 2020). The author also listed the median annual salaries for these four careers ranging from $52 \mathrm{~K}$ to $94 \mathrm{~K}$, while the employment number of all of them expect the multimedia artists will suffer a $-4 \%$ decrease in the next ten years. Many articles presented different views on the income of artists (Miller \& Cuntz, 2018; Dowd, 2020) who often hold a salaried job while doing artwork as a freelancer.

\subsection{New Values and Perspectives in the Cryptocurrency Market}

Digital artwork made recent highlights with the integration of the cryptocurrency market that already tops 2 trillion and is still rising dramatically. A pure digital artwork sold a record 69 million in a recent auction (Moscufo, 2021). A brand-new industry in which digital artworks can be verified authentic through the blockchain, such as the non-fungible tokens (NFTs). Through NFTs, digital artworks can be traded as assets. Therefore, the values of digital artworks are determined by trading or auctions based on the market values using digital currencies. It provides a potentially lucrative avenue for digital artists to generate rev- 
enues since they will receive their share each time their digital artwork is traded online.

All the findings from the literature review promote this study that uses a new method to investigate the impact of the rising popularity of digital art on the job market for artists today.

\section{Method}

Millions of jobs are created every year. Instead of using published (delayed) data for my study, public, online data were collected to reveal how the increasing popularity of digital art impacts the job market for artists today. One of the most common ways for job seekers to uncover employment opportunities is through job search websites. There are many online job boards, social media channels, networking groups, and staffing company websites to collect data. Based on several recommendations (Polner, 2021; Half, 2021), this study selected four major national job search and recruiting websites (LinkedIn, Glassdoor, Indeed, and Monster) to collect data that can represent the size of the current artist job markets. This study also used job functions and industries to indicate whether or not a given artist job can be viewed as a digital artist job. If an artist's job function is information technology (IT), computing, or gaming. If an artist's job is created in the IT, networking, or gaming industries, it is very likely to be a digital art job. The locations of artist jobs were also used to infer the geographic distributions of these artist jobs.

\subsection{Job Market Data Collection and Sampling}

Online public data were collected to reveal the effect of the increasing popularity of digital art is impacting the job market for artists today. Specifically, the active job postings in four major national job search and recruiting websites, LinkedIn, Glassdoor, Indeed, and Monster, were harvested. The first step is to sample the size of the artist job market, and the keyword "artist" was used to identify the artist's job directly. Several advanced options were also used to understand the artist job distributions among industries, job function, and location. The job postings based on the initial posting time of fewer than 30 days were collected. Job market data were collected four times during the study period (January 12 to April 22, 2021). The arithmetic mean and standard deviation were used to represent the datasets.

\subsection{Data Categorization to Identify Digital Art Jobs}

To better understand the job distributions among job functions, the jobs were categorized into four groups: information technology, art/creative, Education, and others. If an artist's job function is IT or computing/gaming, it is very likely a digital art job. The percentage of digital art jobs in the sampled jobs can be calculated.

Four job industries, internet technology, marketing, fashion, and others, were 
also used in the study. If an artist's job is created in the IT, networking, or gaming industries, it is likely a digital art job. The result could confirm the findings from the previous categorization based on job function. For the location, job postings around four major US cities, Los Angeles (art center), New York (art center), San Francisco (tech center), and Seattle (tech center), were collected. The geographic distributions of these digital artist jobs can reveal whether the artist jobs cluster around these cities. Finally, this study also looks into the salary information and group them into two groups: $60 \mathrm{~K}+$ (medium paid) and $100 \mathrm{~K}+$ (high paid). This information helps to answer some questions on the salary of digital artists. Table 1 lists the detailed information on the data collection and categorization.

\subsection{Mathematical Formula for Data Analysis}

\section{Artist Job Data Distribution and Categorization}

Data were collected four times during the study period to reduce the observation bias during the data collection and sampling. The samples were treated as random variables with normal distributions. Arithmetic mean and standard deviation were used to represent the distributions of the job data dataset. The arithmetic mean is the simple average of a list of numbers. In statistics, it measures the central tendency of a probability distribution along median and mode. The standard deviation is a measure of the amount of variation of a set of values. A low standard deviation indicates that the values tend to be close to the mean of the set, while a high standard deviation indicates that the values are spread out over a wider range. The arithmetic mean and standard deviation are calculated using the following formula.

$$
\mu=\frac{1}{N}\left(\sum_{i=1}^{N} x_{i}\right) \text { and } \sigma=\sqrt{\frac{1}{N}\left(\sum_{i=1}^{N}\left(x_{i}-\mu\right)\right)}
$$

where $N$ is the sample size and $x_{i}$ is observation data.

This study also investigates the temporal variation of the artist job market. To deal with discrepancy in sampled data from different resources for artist job trendline analysis, a mean normalization was used to scale the range of data around their means to enable trendline analysis with the data from different resources. The mathematical formula for the mean normalization is

$$
x^{\prime}=\frac{x-\text { average }(x)}{\max (x)-\min (x)}
$$

where $x$ is an original value, $x^{\prime}$ is the normalized value.

Table 1. The categorization and groups used for data collection and sampling.

\begin{tabular}{ccccc}
\hline Job function & Information Technology & Art/Creative & Education & Others \\
\hline Industry & Information Technology & Marketing and Media & Fashion/Design & Others \\
Location & Los Angeles & New York & San Francisco & Seattle \\
Salary & $60 \mathrm{~K}+$ & $100 \mathrm{~K}+$ & & \\
\hline
\end{tabular}




\section{Results and Analysis}

The first website used was LinkedIn, which is the world's largest online professional network. LinkedIn provides a comprehensive way to find the right job or internship, connect and strengthen professional relationships. LinkedIn can be accessed from a desktop, LinkedIn mobile app, mobile web experience, or the LinkedIn Lite Android mobile app. In this study, data from LinkedIn were collected to draw some conclusions, then extra data from other websites were used to support or against these findings from the LinkedIn data. The keyword "artist" was used to identify artist jobs. In the study period (January 12 to April 22, 2021), the average number of artist postings on LinkedIn (without salary restriction) is around 22,500, with a standard deviation of 3400. Two salary restrictions $(60 \mathrm{~K}+$ and $100 \mathrm{~K}+$ ) were used to filter the jobs within LinkedIn further. After that, these jobs were categorized based on their job function, industries, and location. The results were listed in Table 2.

As shown in Table 2, on average, there are around $75 \mathrm{~K}$ artist jobs (salary $>60$ $\mathrm{K})$ advertised within LinkedIn in the period of study from early January to late April. Among these jobs, around 32\% (2395 out of 7515) are higher-paid jobs (salary $>100 \mathrm{~K}$ ). It is also reasonable to conclude that most artist jobs in LinkedIn are related to digital art. By looking into the job distribution among job functions, it is also clear that the majority (66\%) of the artist jobs (4960 out of 7515) are from IT, followed by $24 \%$ (1765 out of 7515) from the general Art/Creative job function. Less than $5 \%$ of artist jobs were associated with Education, including teachers in schools or academic institutes. Around 8\% - 10\% of artist jobs are associated with other job functions, such as engineering, manufacturing. It was evident that most artist jobs with a salary over $65 \mathrm{~K}$ are associated with IT functions. LinkedIn has a solid reputation for attracting IT jobs; therefore, most job postings, even the artist jobs, in LinkedIn are related to IT. When looking into the data distribution of these jobs (salary $>60 \mathrm{~K}$ ) among industries, most artist jobs (around 65\%) are created in the IT sectors, 20\% from Marketing/Entertainment sector, around 5\% from Fashion \& Design industries, the remaining around $15 \%$ from other sectors. To better present these job distributions along with job functions and industries, pie charts have been created and illustrated (Figure 1).

At last, the artist jobs did not cluster around major IT cities, such as San Francisco or Seattle, nor around the economic centers, such as New York City or Los Angeles. The jobs (salary $>60 \mathrm{~K}$ ) among these four big cities take less than $33 \%$, while the percentage of these jobs (salary $>100 \mathrm{~K}$ ) among four big cities is less than 25\%. Therefore, the artist jobs are distributed across the US (Figure 2).

The standard deviation associated with the arithmetic means ranges from $20 \%-40 \%$, representing significant data variation among these collected data. Typically, many new jobs are posted in the Jan-April period. This year, the job market dynamics are also heavily influenced by the recovery from the pandemics. 
Table 2. Artist Job posting in LinkedIn within the past 30 days from observation dates (with four data collections from February 12, 2021, to April 20, 2021)*.

\begin{tabular}{cccccc}
\hline Salary & \multirow{2}{*}{ Total Jobs } & \multicolumn{4}{c}{ Job functions } \\
\hline & & IT & Art/Creative & Education & Others \\
\hline $100 \mathrm{~K}+$ & $2395(459)$ & $1655(319)$ & $576(154)$ & $39(13)$ & $126(54)$ \\
$60 \mathrm{~K}+$ & $7515(1708)$ & $4960(1321)$ & $1765(527)$ & $374(94)$ & $417(369)$ \\
\hline \multicolumn{5}{c}{} & \multicolumn{5}{c}{ Industries } \\
\hline $100 \mathrm{~K}+$ & $2395(459)$ & $1785(299)$ & $441(94)$ & $56(9)$ & $113(68)$ \\
$60 \mathrm{~K}+$ & $7515(1708)$ & $4732(1270)$ & $1469(330)$ & $430(59)$ & $884(347)$ \\
\hline & & & Marketing \& & Fashion \& Design & Others \\
\hline & & San Francisco & New York & Los Angeles & Seattle \\
\hline $100 \mathrm{~K}+$ & $2395(459)$ & $324(71)$ & $175(80)$ & $154(45)$ & $221(36)$ \\
$60 \mathrm{~K}+$ & $7515(1708)$ & $465(108)$ & $494(211)$ & $421(121)$ & $375(70)$ \\
\hline
\end{tabular}

* The first value is an arithmetic mean, and the second value (within the bracket) is one standard deviation.
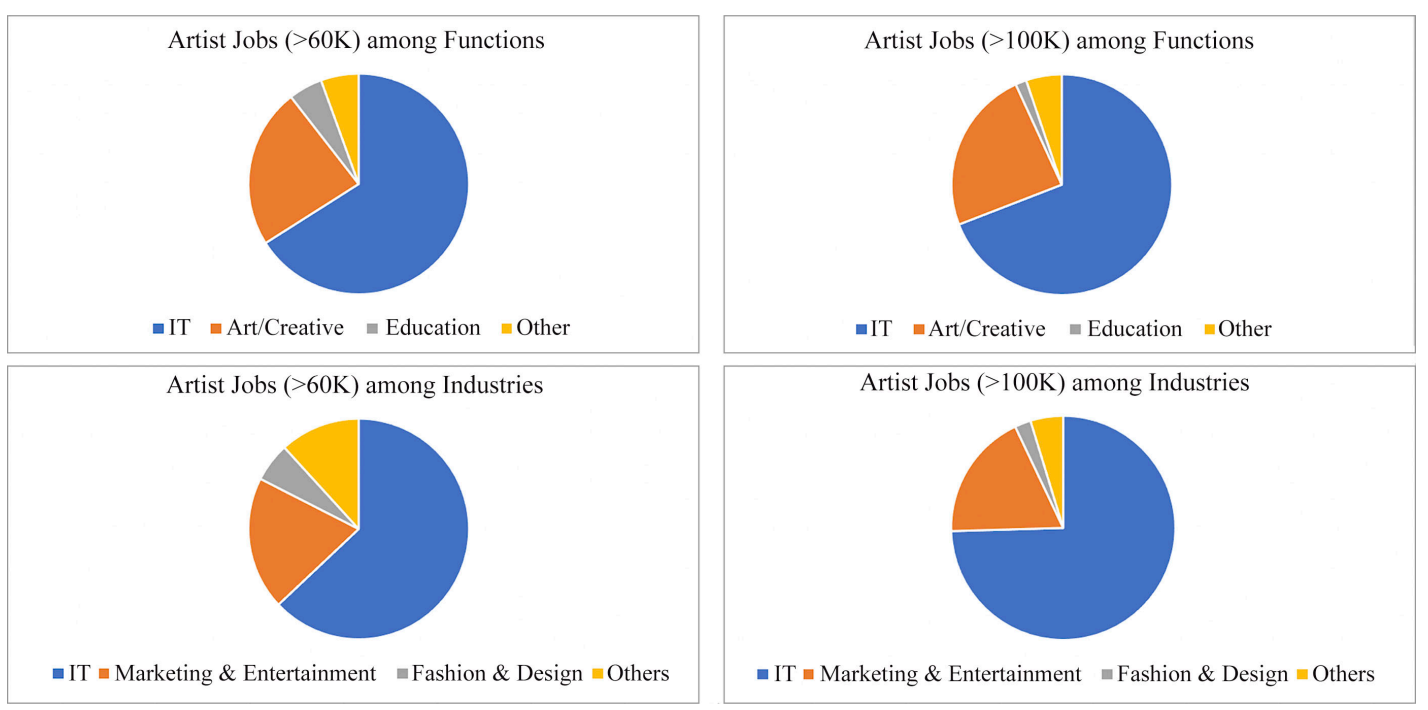

Figure 1. Artist job distribution among job functions and industries (with four data collections from LinkedIn: February 12 to April 20, 2021).
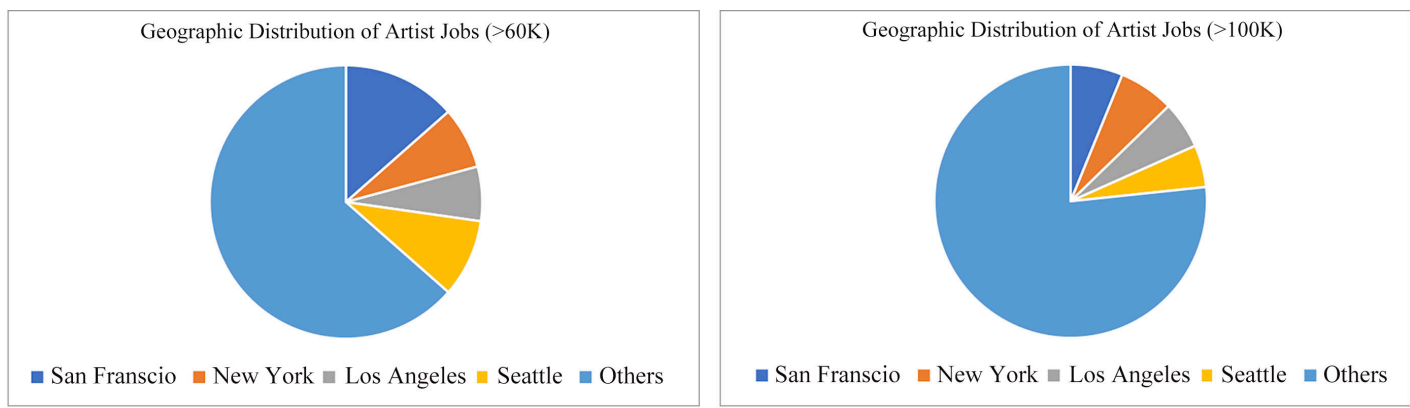

Figure 2. Geographic distribution of artist jobs around four large US cities (with four data collections from LinkedIn). 
The second website used is Glassdoor.com, another large job recruiting site. Glassdoor was built on the foundation of increasing workplace transparency and offers millions of the latest job listings. Most significant features, Glassdoor provides rich information on company reviews, salary reports. All of the information on Glassdoor is shared by actual employees. Unlike LinkedIn, Glassdoor does not allow users to search for jobs with several predefined salary ranges. Therefore, the keyword "artist" was used to search the jobs posted in the last 30 days with a salary larger than $59 \mathrm{~K}$. The sampled results (collected four times from February 12 to April 20, 2021) are listed in Table 3 and Figure 3.

As shown in Table 3, the search for artist jobs returned around 2350 hits. The most significant portion of artist jobs (40\%) comes from two industries, IT/Internet and Marketing/Entertainment, in which the majority are digital jobs. Again, the artist jobs do not cluster around major cities, as the jobs around these big cities only take less than $25 \%$ of the total jobs. These are similar to what has been found from LinkedIn data. The job distributions along industries as well as the geographic distributions are presented as pie charts in Figure 3. Again, the standard deviations indicate significant variations among collected data.

Indeed.com is the third website used for data collection. Several keyword combinations (listed in the first row of Table 4) were used with an annual salary of over $60 \mathrm{~K}$. It only provides an option to search for a job posting within 14 days. The data from Indeed.com supports two conclusions: 1) the majority of artist jobs (around 60\%, or 3800 out of 6300) are related to digital art and technology, and 2) the jobs are distributed across the US, and the majority of artist jobs did not cluster in several major cities.

Monster.com is the fourth website used for the research. It is a 25 -year-old job search engine. Monster.com does provide advanced search options as LinkedIn and Glassdoor, but the data collections are not as complete as the other two websites. Therefore, these data were used to support some of the previous conclusions from other datasets.

The data collected from Monster.com was counterintuitive. The job hits with combined keywords ("artist" and other keywords) along with a salary restriction over $60 \mathrm{~K}$ returned more hits than the search with the single keyword "artist". Therefore, the "digital artist" keyword was used to identify digital artist jobs in the nation. The job locations among these job hits were analyzed to understand the geographic distribution of these jobs (Table 5). Again, the jobs are distributed across the US, and most artist jobs did not cluster around major cities.

At last, the total job postings on these four websites during the research period (Jan-April) were used to identify the temporal variation with these job postings with a salary larger than $60 \mathrm{~K}$ (Figure 4). Large temporal variations were observed (left panel of Figure 4), except for the data from Glassdoor. The total number of artist jobs in the US during the research period is much bigger than the estimations from the previous reports (Miller \& Cuntz, 2018; Dowd, 2020). Many artist jobs with a solid connection to IT and digital art came with a medium to a high salary. Most importantly, a significant increase of the digital artist 
jobs was observed at the end of the study period (right panel of Figure 4), which was not a surprise since the US economy is expanding as we are recovering from the Pandemics. The majority of the new jobs (with a salary over $60 \mathrm{~K}$ ) are digital art jobs. Therefore, the increasing popularity of digital art has had a big impact on the current job market for artists.
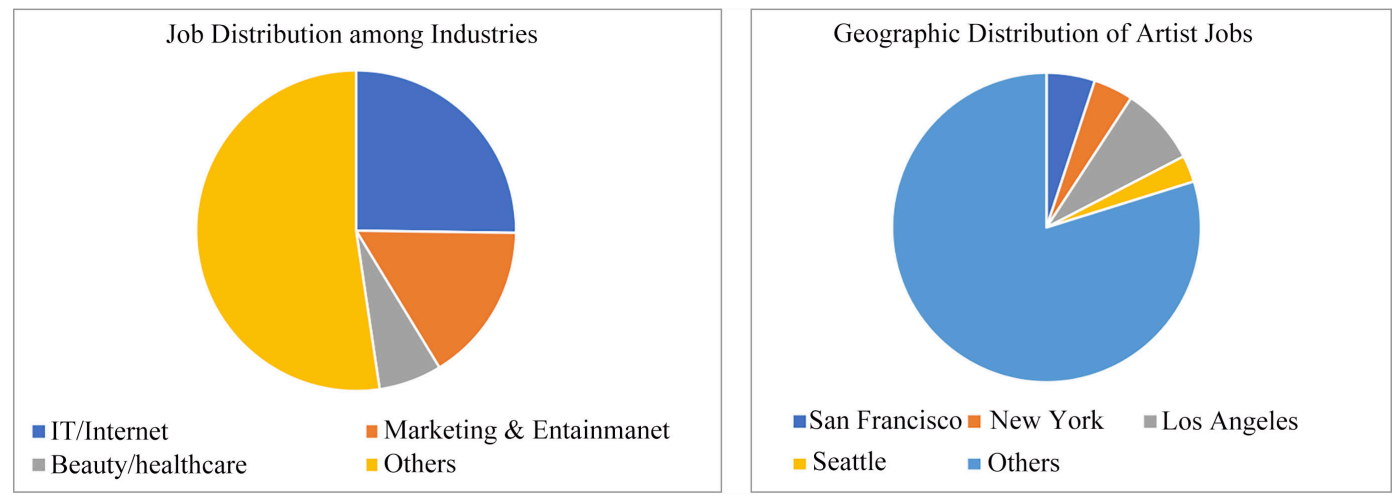

Figure 3. Artist job distribution among industries and big cities (with four data collected from Glassdoor with a salary restriction of over $60 \mathrm{~K}$ ).
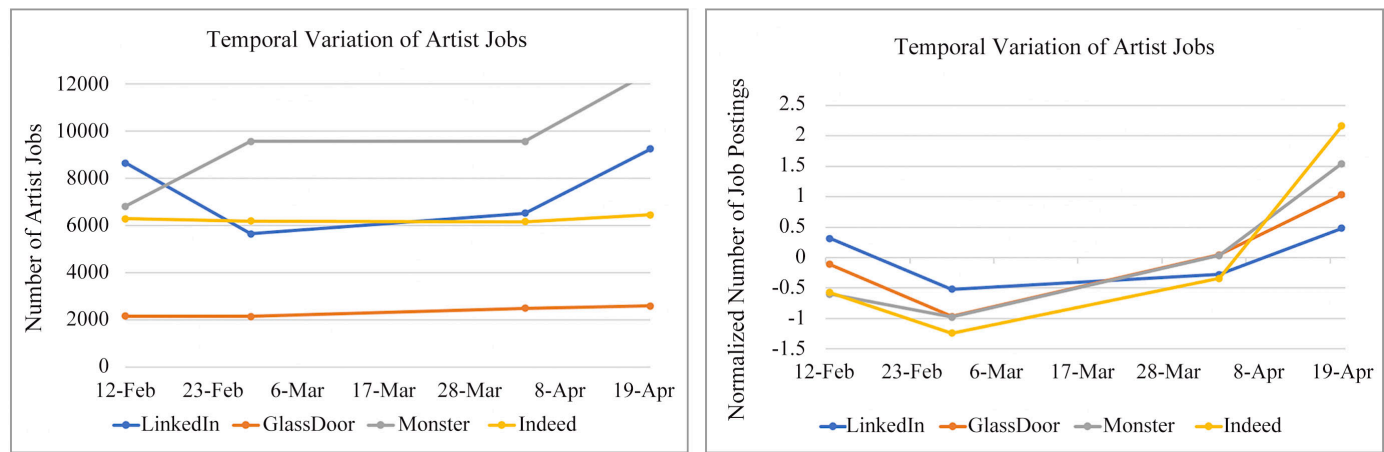

Figure 4. The temporal variation of the artist job posting data from four websites (the left panel shows the raw data and the right panel shows the normalized data).

Table 3. Artist job distributions among industries (arithmetic mean with one deviation) (with four data collections from Glassdoor.com: February 12 to April 20, 2021).

\begin{tabular}{ccccc}
\hline Total (national) & IT/Internet & Marketing \& Entrainment & Fashion \& Design & Others \\
\hline $2343(227)$ & $591(192)$ & $376(228)$ & $149(188)$ & $1227(397)$ \\
\hline Total (national) & San Francisco & New York & Los Angeles & Seattle \\
\hline $2343(227)$ & $119(66)$ & $97(20)$ & $191(91)$ & $66(38)$ \\
\hline
\end{tabular}

Table 4. Artist job postings (arithmetic mean with one deviation) from Indeed.com.

\begin{tabular}{ccccc}
\hline Artist & Digital artist & Graphic artist & Game artist & Others \\
\hline $6297(113)$ & $1371(380)$ & $1435(495)$ & $930(967)$ & $1978(1918)$ \\
\hline Nationwide & SF & NY & Los Angeles & Seattle \\
\hline $6297(113)$ & $97(57)$ & $222(84)$ & $154(79)$ & $48(24)$ \\
\hline
\end{tabular}


Table 5. Artist job search results (arithmetic mean with one deviation) from Monster.com.

\begin{tabular}{ccccc}
\hline Nationwide & San Francisco & New York & Los Angeles & Seattle \\
\hline $8851(2818)$ & $501(358)$ & $935(726)$ & $398(269)$ & $358(193)$ \\
\hline
\end{tabular}

\section{Conclusions and Discussions}

Based on the data collected from the four websites in the study period (Jan-April 2021), the following conclusions on artist jobs are made:

1) The job market for digital artists is not small. The average number of job postings on LinkedIn without salary restriction is around 22,500 and a standard deviation of 3400 . This number is much larger than the estimation from the $\mathrm{Bu}$ reau of Labor Statistics' labor report for 2019-2029.

2) On average in LinkedIn, around $75 \mathrm{~K}$ artist jobs with a salary of over $60 \mathrm{~K}$ are available. Among these job postings, around 33\% are higher-paid jobs with a salary of over $100 \mathrm{~K}$. Most of these artist jobs are related to digital art. The job function data analysis showed that the majority (65\%) of these artist jobs are related to information technology. The most popular digital art jobs are related to graphics.

3) Most (85\%) of these artist jobs with a salary over $60 \mathrm{~K}$ are digital artist jobs and are created in information technology, marketing, and entertainment industries.

4) These jobs are created across the nation, not just clustered around major cities, such as New York City and Los Angeles, or major technical centers, such as San Francisco and Seattle.

As observed in all four websites, the total number of artist jobs (without salary restrictions) in the US during the research period is much larger than the number retrieved from the previous reports (Writers, 2020; Dowd, 2020). First, the number of artist jobs, especially digital artist jobs, is increasing over time, not decreasing. Second, these artist jobs are decent and well-paid, which means that there is no need for a second job. Specifically, the majority (65\%) of the artist jobs (with a salary of over $60 \mathrm{~K}$ ) are related to IT and digital art. Also, $85 \%$ of the artist jobs are created in the IT and marketing/entertainment industries. Therefore, the increasing popularity of digital art has a significant impact on the current job market for artists, especially these high-paid jobs.

There are some limitations of this study. Due to the time restriction, this study used online data from job recruiting sites in a short period (around three months) to investigate the job market for digital artists. A longer-term observation (at least a couple of months) is needed to confirm the size of artist job markets and job distribution across sectors and functions. A longer-term observation can also provide information on possible seasonal job fluctuation and growth trends. This study only used the data from several national job recruiting sites. The job posting on these websites is not free, and many employers use many other resources, such as newsletters and social media. Future studies could develop computer programs (with web technologies) to harvest data through a collection 
of online job resources, such as national job recruiting sites, social media, blogs, and key career development sites.

It will be fascinating to understand the impact of new emerging opportunities/technologies (such as non-fungible tokens and cryptocurrencies) on the job market for digital artists. I would like to track and analyze the user and trade information from several open digital art marketplaces, such as OpenSea.io, Rarible, Foundation, and e-markets on eBay and Instagram.

\section{Acknowledgements}

The author also thanks Mrs. Kelly Loveday at Farragut High School for her valuable comments on manuscript preparation.

\section{Conflicts of Interest}

The authors declare no conflicts of interest regarding the publication of this paper.

\section{References}

Agyeman, C. A. (2014). Artists' Perception of the Use of Digital Media in Painting. ProQuest LLC. https://eric.ed.gov/?id=ED567903

Anton, G. (2020, December 7). 15 Types of Digital Art to Consider. Architecture Lab. https://www.architecturelab.net/types-of-digital-art

Bessette, J. (2018). The Machine as Art (in the 20th Century): An Introduction. Arts, 7, 4. https://doi.org/10.3390/arts7010004

Butcher, A. J. (2017). The Growing Popularity of the Digital Arts Movement Put into Context by LACDA's Rex Bruce.

https://www.huffpost.com/entry/the-growing-popularity-of_b_9287218

Didenko, I. (2018, January 15). The Evolution of Art: Will Pixels Replace Paint? Art Acacia Gallery.

https://www.artacacia.com/blogs/posts/the-evolution-of-art-will-pixels-replace-paint

Dowd, M. (2020, September 11). How Much Money Do Visual Artists Make in a Year? Work. https://work.chron.com/much-money-visual-artists-make-year-10538.html

Edmonds, E. (2019) Communication Machines as Art. Arts, 8, 22. https://doi.org/10.3390/arts8010022

Half, R. (2021). 25 Best Job Search Websites in 2021. 25 Best Job Search Websites. https://www.roberthalf.com/blog/job-market/10-best-job-search-websites

Heck, C. (2017). The Rise of Digital Art. The Broadcaster. https://hhsbroadcaster.com/4399/ane/the-rise-of-digital-art

Iyengar, S. (2011, June 27). Artist Employment Projections through 2018. National Endowment for the Arts. https://www.arts.gov/sites/default/files/103.pdf

Miller, A., \& Cuntz, A. (2018). Unpacking Predictors of Income and Income Satisfaction for Artists. https://www.wipo.int/edocs/pubdocs/en/wipo_pub_econstat_wp_50.pdf

Moscufo, M. (2021, March 11). Digital Artwork Sells for Record \$69 Million at Christie's First NFT Auction. NBCNews.com.

https://www.nbcnews.com/business/business-news/digital-artwork-sells-record-60-mil $\underline{\text { lion-christie-s-first-nft-n1260544 }}$ 
Paul, C. (2008). Digital Art (2nd ed.). Thames \& Hudson.

Polner, E. (2021). The 10 Best Job Search Websites of 2021. The Balance Careers. https://www.thebalancecareers.com/top-best-job-websites-2064080

Sanders, K. (2018). Why Digital Art Is Better than Traditional: The Painted Kat. The Painted Kat|Dream! Create! Inspire! Be Epic!

http://paintedkat.art/why-digital-art-is-better-than-traditional-art

Schukei, A. (2019, May 10). Digital Vs. Traditional Art: Is One Better than the Other? The Art of Education University.

https://theartofeducation.edu/2019/05/29/digital-vs-traditional-art-is-one-better-than-t he-other

Writers, S. (2020). Careers in Art. Affordable Colleges Online. https://www.affordablecollegesonline.org/degrees/art-schools/careers

Zagrobelna, M. (2017, November 17). How Is Digital Drawing Different from Traditional Art? Medium.

https://medium.com/sketchbook-in-perspective/how-is-digital-drawing-different-from -traditional-art-8204e5954d6b 